\title{
Francisco Ayala y José Medina Echavarría: entre el exilio y la sociología latinoamericana
}

\author{
Francisco Ayala and José Medina Echavarría: between exile and \\ sociology in Latin America
}
Francisco Ayala e José Medina Echavarría: entre o exílio e a sociologia latino-americana

Luis Alberto Escobar*

\section{A Alberto y a Juan por demostrar diariamente que otros mundos académicos son posibles}

\begin{abstract}
RESUMEN
El trabajo se articula alrededor de las trayectorias de los exiliados españoles Francisco Ayala y José Medina Echavarría en el periodo 1939-1949 en Latinoamérica, a fin de mostrar el papel que jugaron en la incorporación y/o relectura de algunos autores fundamentales de la tradición sociológica germánica y anglosajona, en particular de Karl Mannheim, Max Weber, Ferdinand Tönnies y John Dewey. Siguiendo los intercambios de los sociólogos españoles, uno en México y otro en Buenos Aires, se destacan las continuidades de una relación a la distancia, así como el rol y las apuestas de ambos en la edición de libros y circulación dentro de los espacios académico-intelectuales, el campo editorial y cultural. Se observa, asimismo, una red vincular de correspondencias, aclimatación e incorporación de preocupaciones intelectuales y políticas, de productos y proyectos de los sociólogos que va conformando un corredor entre México y Buenos Aires, y, a su vez, este se ramifica de acuerdo a los espacios y agentes con que uno y otro se relacionan. Entonces, desde estos trazos de las trayectorias intelectuales y su red vincular, se aborda el aporte de estos
\end{abstract} Palabras clave: difusión intelectual, exilio español, Francisco Ayala, José Medina Echavarría, sociología en Latinoamérica.

Doctor en Estudios Sociales de América Latina, Universidad Nacional de Córdoba. Universidad Nacional del Litoral, Santa Fe, Argentina. E-mail: 1.al.escobar@gmail.com 
dos exiliados españoles en el proceso de renovación disciplinar de la sociología en la región.

\begin{abstract}
The work is structured around the trajectories of the Spanish exiles Francisco Ayala and José Medina Echavarría in Latin America during the period 1939-1949, in order to show the role they played in the incorporation and/or rereading of some fundamental authors of the Germanic and Anglo-Saxon sociology tradition, in particular: Karl Mannheim, Max Weber, Ferdinand Tönnies and John Dewey. Following the exchanges of the Spanish sociologists, one in Mexico and the other one in Buenos Aires, the continuity of their relationship at a distance is highlighted, as well as their role, edition of books and circulation within the academic-intellectual spaces and the editorial and cultural field. Also observed is a network of correspondence, acclimatization and incorporation of intellectual and political concerns, of products and projects of sociologists to form a corridor between Mexico and Buenos Aires, that in turn branches out according to the spaces and agents to which they relate. Then, based on this tracing of the intellectual trajectories and the network that connects them, the contribution of these two Spanish exiles in the process of the disciplinary renewal of sociology in the region is addressed.
\end{abstract}

\section{RESUMO}

O trabalho é estruturado em torno das trajetórias dos exilados espanhóis Francisco Ayala e José Medina Echavarría no período de 1939-1949 na América Latina, a fim de mostrar o papel que tiveram na incorporação e/ou releitura de alguns autores fundamentais da tradição sociológica germânica e anglo-saxônica, em particular Karl Mannheim, Max Weber, Ferdinand Tönnies e John Dewey. Seguindo os intercâmbios dos sociólogos espanhóis, um no México e outro em Buenos Aires, destaca-se a continuidade de uma relação à distância, assim como o papel e as apostas de ambos na edição de livros e na circulação dentro dos espaços acadêmico-intelectuais e nos campos editorial e cultural. Observa-se também uma rede de correspondência que os vincula, além da aclimatação e incorporação de preocupações intelectuais e políticas, de produtos e projetos dos sociólogos que formam um corredor entre o México e Buenos Aires, e que, por sua vez, se ramifica de acordo com os espaços e agentes com os quais um e outro se relacionam. Então, a partir destes traços
Keywords: intellectual diffusion, Spanish exile, Francisco Ayala, José Medina Echavarría, sociology in Latin America.

Palavras-chave: difusão intelectual, exílio espanhol, Francisco Ayala, José Medina Echavarría, sociologia na América Latina. 
das trajetórias intelectuais e de sua rede de vínculos, é abordada a contribuição destes dois exilados espanhóis no processo de renovação disciplinar da sociologia na região. 


\section{Introducción}

La propuesta pone el acento en la trayectoria latinoamericana de Francisco Ayala (Granada, 1906 - Madrid, 2009), y, de su compañero de ruta, José Medina Echavarría (Castellón de la Plana, 1903 - Santiago de Chile, 1977). Ambos en su juventud se perfeccionan mediante becas de estadía en los principales centros europeos de la época para modernizar la disciplina sociológica en España ${ }^{2}$, acción que comienzan a llevar a cabo en su país como jóvenes catedráticos universitarios e incipientes intelectuales y, asimismo, como funcionarios de la Segunda República española a principios de la década de 1930. Con el estallido de la Guerra Civil y la posterior derrota del bando republicano, estos jóvenes se ven obligados a desarmar sus recientes itinerarios y proyecciones para comenzar el exilio al otro lado del Atlántico. Hacia 1939, Medina logra asentarse en México y Ayala ingresa a Argentina. Cada uno, en esos nuevos espacios, busca retomar parte de sus proyectos inconclusos en la península, adaptados a las condiciones locales a la vez que tratan de comprender lo sucedido. Desde este punto, el artículo centra su interés en ciertos aportes que Ayala y Medina harán a la sociología latinoamericana, en tanto mediadores, productores y difusores de algunos autores, obras y temáticas propias de este campo.

Es importante considerar que los procesos de corte intelectual no se reducen solo a instituciones, que si bien constituyen un espacio de profunda influencia, no logran captar ni definir en su totalidad; por eso aquí se pretende ampliar la mirada y poner el acento en los intercambios concretos, en las producciones y circulaciones de autores y obras, en las búsquedas y apuestas que van entretejiendo un corredor de ideas por el que se vinculan y vehiculizan agentes, espacios institucionales y culturales, dando forma a constelaciones en un intercambio de

2 Es de destacar que tanto Medina como Ayala realizaron viajes de estudio a Alemania, tránsito casi obligado de los jóvenes universitarios. Es coincidente, también, que fueron becados por la Junta para la Ampliación de Estudios e Investigaciones Científicas (JAE), institución clave en la renovación científico-intelectual y vincular:

La JAE promovió un sistema de pensiones para que los estudiantes, académicos y profesionales becados cursaran estudios de perfeccionamiento y de especialización en el exterior. Ante la falta de docentes y de especialistas la movilidad académica se convirtió en una forma de incrementar el nivel nacional de las disciplinas científicas y de actualizarlas en cuanto a la importación de nuevos métodos, bibliografías, pedagogías o prácticas. (Morales Martín, 2017, p. 44). 
materialidades, proyectos y aclimataciones entre México y Argentina (Morales Martín, 2014).

Durante el período en que Ayala logra asentarse en Argentina (1939-1949), comienza de forma temprana un trabajo de difusión intelectual poco conocido dentro de la sociología, el que, además, a través de sus múltiples prácticas e intervenciones, incide también en una renovación disciplinar de la sociología en la región más vasta. Esta trayectoria, enlazada a la más estudiada experiencia de José Medina Echavarría en México y a la colección de sociología que este dirige en el Fondo de Cultura Económica, permite reponer los vínculos entre ambos y establecer relaciones, comparaciones, e incluso algunas contraposiciones y complementariedades entre los dos españoles y su trabajo como difusores intelectuales plasmado en un diálogo regional.

En esta exploración a través de los catálogos bibliográficos, el mundo impreso y la difusión en términos amplios, puede observarse un nuevo giro en la recepción e interpretación del pensamiento alemán -que ya constituía ser una referencia en los sociólogos locales-, pero también del mundo anglosajón y de la teoría sociológica en general. Esto, en gran medida, es facilitado por la hibridación causada por el exilio. Desde esta perspectiva, se busca, entonces, aportar luz sobre el papel que jugaron Medina y Ayala en esta difusión, en el debate y también la incorporación - a través de sus propias obras, traducciones y diferentes acciones- de algunos autores fundamentales de la tradición sociológica germánica y anglosajona en el medio latinoamericano.

\section{Presencias, materialidades y vinculaciones de Ayala y Medina en la región}

En su temprano arribo a México en 1939 Daniel Cosío Villegas le confía a José Medina Echavarría la dirección de la Sección de Obras de Sociología (SOS) de la editorial Fondo de Cultura Económica (FCE). Este último con la asistencia de Cosío y un amplio grupo editor emprende la publicación de una biblioteca que se irá posicionando como icónica para la sociología y, en general, para las ciencias sociales de habla hispana. Laura Moya López (2013) da cuenta de los números en relación a los años 1939-1946 y de la vinculación de Medina como difusor y transmisor: 
La Colección de Sociología iniciaba en 1939 con dos títulos; en 1940 con tres; en 1941 con diez títulos nuevos; en 1942 con ocho más. Para 1943 se integraron seis nuevos libros; en 1944 siete; en 1945 seis, y en 1946, otros seis títulos. La selección de los primeros títulos, de 1939 a 1943, respondía en buena medida a la necesidad de proveer las bibliografías solicitadas por Medina Echavarría en sus cursos. (p. 93).

En los años mencionados, Medina realiza tres traducciones para la SOS, estas son:

- Max Weber (1944). Economía y sociedad (editada por primera vez en cuatro volúmenes, en cuya traducción Medina Echavarría -que coordinó la traducción y la edición general- también prologó el primer volumen).

- Karl Mannheim (1944). Diagnóstico de nuestro tiempo.

- Harry Alpert (1945). Durkheim.

Ya en 1949, desde Puerto Rico, colabora con Julián Calvo y Tomás Muñoz en la traducción y revisión del Diccionario de Sociología de Henry Pratt Fairchild.

Además de las traducciones Medina comienza a publicar sus propios títulos en la región. En el período abordado, estos son:

- (1940) Panorama de la sociología contemporánea. La Casa de España en México, México.

- (1941) Sociología: Teoría y técnica. FCE, México.

- (1943) Responsabilidad de la inteligencia: Estudios sobre nuestro tiempo. FCE, México.

- (1945) Consideraciones sobre el tema de la paz. Banco de México, México.

Por otra parte, vale destacar que Medina tuvo una activa participación en publicaciones periódicas mexicanas como Cuadernos Americanos, El Trimestre Económico, Revista Mexicana de Sociología, Noticiero Bibliográfico. Varios de los artículos publicados en estos espacios son recogidos en sus libros de forma parcial o total. 
Entre 1939 y 1946, se cuentan publicados en la SOS más de 40 títu$\operatorname{los}^{3}$, un numeroso catálogo cuyo espectro de temas, autores, tradiciones teóricas y disciplinares también es amplio. Siguiendo el planteo de Juan Morales Martín (2017), en la SOS

se tradujeron y se publicaron manuales básicos y fundamentales para entender la sociología (...) Además se inició una serie monográfica sobre los grandes sociólogos contemporáneos para reconocer la importancia científica de autores (...) Se publicaron algunas obras bajo un enfoque multidisciplinar (...) hubo lugar para la aparición de títulos de autores latinoamericanos (...) Pero, sin duda, la característica que definió a esta sección de sociología del Fondo de Cultura Económica fue la traducción de los autores alemanes. (pp. 119-120).

Sin duda que el conocido catálogo sociológico del FCE comenzó a producir un gran foco de difusión. Incluso se observa que en este temprano momento se publican algunos de "los grandes textos de lo que podemos identificar como la gran tradición sociológica" (Blanco, 2006a, p. 113), los cuales a través de esta colección de manera bastante temprana "ya están disponibles en español" (Blanco, 2006a, p. 113). Aunque la gran tradición sociológica está en permanente construcción, y sin duda que en el contexto de los años cuarenta ese listado no coincide de manera plena con la actualidad, dado que hay autores y obras que quizá no reconoceríamos como participantes de la tradición, e incluso otros que todavía no se habían posicionado en tal corpus en dicho contexto. Si hacemos un repaso rápido de los autores publicados por el FCE, bajo la dirección de Medina, vemos que se encuentran Max y Alfred Weber, Ferdinand Tönnies, Karl Mannheim, Auguste Comte, Florian Znaniecki, Thorstein Veblen, nombres que, con más o menos presencia, encontramos hoy en la gran tradición sociológica. Sobre esos pocos nombres advertimos que se efectiviza, a través de la mayoría, la afirmación de Morales Martín (2017) acerca de la característica definitoria - aunque no exclusiva- de la SOS como una gran traductora de la sociología alemana.

La consulta al Catálogo histórico 1934-2009 (Fondo de Cultura Económica, 2009) referencia un total de 44 libros en este período, número que no coincide con lo expuesto por Laura Moya López — quien referencia 48 títulos-debido, quizás, a la inclusión de algunos títulos que luego han sido reclasificados dentro de otras colecciones. 
Un par de años después del inicio de la SOS del FCE en Argentina hacia 1941, se presenta la primera colección sociológica local: hablamos de la denominada Biblioteca Sociológica de la editorial Losada, cuya dirección estaba a cargo de Francisco Ayala. Allí se editaron los siguientes títulos:

- Georges Gurvitch (1941), Las formas de la sociabilidad. Ensayos de sociología (traducción de Francisco Ayala).

- Morris Ginsberg (1942). Manual de sociología (traducción de José Medina Echavarría).

- Hans Freyer (1944). La sociología, ciencia de la realidad. Fundamentación lógica del sistema de la sociología (traducción y prólogo de Francisco Ayala).

- Robert M. MacIver (1944). Comunidad. Estudio sociológico, intento de establecer la naturaleza y leyes fundamentales de la vida social (traducción de Jesús Prados Arrarte).

- José Ingenieros (1946). Sociología argentina (notas de Aníbal Ponce).

- Wilhelm Pinder (1946). El problema de las generaciones en la historia del arte de Europa (sección sociologías especiales; traducción de David J. Vogelmann y nota de Francisco Ayala).

- Giséle Freund (1946). La fotografía y las clases medias en Francia durante el siglo XIX. Ensayo de psicología y de estética (sección sociologías especiales; traducción de María Luisa Navarro Margati).

- Ferdinand Tönnies (1947). Comunidad y sociedad (traducción de José Rovira Armengol).

Una primera comparación que asemeja la colección de Medina a la de Ayala es que los nombres más fuertes están relacionados con la tradición sociológica alemana, lo que se refleja en la cantidad de obras traducidas dentro de la colección, así como una enorme diferencia es la cantidad total de títulos publicados en un período temporal análogo. Una respuesta a esta diferencia podemos buscarla en la comparación con el tipo de emprendimiento editorial en la que se edita cada colección.

El FCE tiene una impronta de ciencias sociales desde su mismo inicio como catálogo de economía que con este tipo de ramificaciones, 
luego fortalecería y profundizaría la línea fundacional. Es "una editorial creada para mejorar el conocimiento de la economía en México, que pronto se transformó en una editorial académica dedicada a las ciencias sociales y las humanidades" (Garciadiego, 2016, p. 62). En tanto que, si bien tuvo presencia en el campo de las sociales y humanas, la centralidad de Losada estuvo colocada en la literatura, que será la que la catapultará en el mercado cultural hispano-parlante aunque desde un perfil un tanto ecléctico y universalista (Diego de, 2014). Por otro lado, mientras que el FCE está vinculado al Estado mexicano, ya que se trató desde su origen y evolución de una "empresa autónoma subsidiada con fondos públicos" (Sorá, 2010, p. 545), la editorial Losada es un emprendimiento totalmente privado.

Es también preciso aclarar en el caso de Ayala que la cantidad de títulos publicados durante el período 1939-1949 se amplía si se suman el armado de colecciones específicas y dispersas de ediciones que exceden la Biblioteca Sociológica de Losada ${ }^{4}$. Si contamos estos libros, se integran la colección también dirigida por el español: Los Clásicos Políticos editada por Americalee, compuesta de ocho libros; un segundo grupo de publicaciones editadas por fuera de colecciones específicas - y en distintas editoriales - en las que interviene Ayala con diferentes acciones (estudios introductorios, traducción, prólogos), que suman al momento cinco títulos ${ }^{5}$ y, finalmente, en el último grupo sus propios libros en tanto autor, que constituyen siete títulos más dentro del período abordado ${ }^{6}$. Por lo cual, se organiza un catálogo sociológico verte-

\footnotetext{
$4 \quad$ Por supuesto que este corpus se trata de una selección de la bibliografía orientada específicamente en relación a las ciencias sociales, dejando de lado con el recorte, de momento, la obra literaria de Ayala.

En ese marco, podemos mencionar de lo encontrado al momento, ya que es una búsqueda aún activa, (1941) El pensamiento vivo de Saavedra Fajardo. Buenos Aires, Losada (presentado por Francisco Ayala); Bentham (1944) Tratado de los sofismas politicos. Rosario, Ed. Rosario (traducción y prólogo de Francisco Ayala); Georg Simmel (1944) Schopenhauer y Nietzsche. Buenos Aires, Schapire (traducción de Francisco Ayala); Herbert Spencer (1945) El hombre contra el Estado. La Plata, Yerba Buena (traducción de Siro García del Mazo e introducción y notas de Francisco Ayala); y AA.VV. (1945) Jovellanos, su vida y su obra. Buenos Aires, Centro Asturiano (artículo de Francisco Ayala, "Jovellanos sociólogo").

Los títulos propios son (1941) El problema del liberalismo. México, FCE; (1942) Oppenheimer. México, FCE; (1943) Historia de la libertad. Buenos Aires, Atlántida; (1944) Razón del mundo. Un examen de conciencia intelectual. Buenos Aires, Losada; (1944) Los políticos. Buenos Aires, Depalma; (1944) Ensayo sobre la libertad. México, Colegio
} 
brado en diálogos de continuidad que conforman un corpus, con una sumatoria total de 28 títulos (Escobar, 2018).

Asimismo, como se destacó con Medina, Ayala también tuvo una fuerte presencia en diarios y revistas de Argentina y de la región en el período estudiado. A modo de mención colaboró activamente en las páginas de diario La Nación, revista La Ley, Correo Literario, De Mar a Mar, Sur, Argentina Libre, Cuadernos Americanos, revista Universidad, diario El Litoral, Boletín del Instituto de Sociología de la UBA, Cursos y Conferencias del CLES, Revista de las Indias, Revista de Ciencias Jurídicas y Sociales de la UNL, Pensamiento Español, Realidad. Revista de Ideas, Ver y Estimar, Cursos Libres de Portugués y Estudios Brasileños.

\section{Sociología, espacios académicos y culturales}

Una de las dimensiones menos visibilizadas para la institucionalización y/o renovación de un campo disciplinar en los espacios académicos es la de las actividades editoriales y sus agentes a través de las variadas acciones de aclimatación y difusión intelectual. Es por ello que, como venimos exponiendo, la tarea editorial amplia de Ayala y Medina merece una revisión para dar cuenta del abanico de acciones relacionales: la escritura, el comentario (prólogo, estudios preliminares, presentaciones), la traducción, la dirección y asesoría editorial, los vínculos intelectuales y culturales y la difusión en revistas y diarios constituyen un conjunto de actividades que nutrieron un campo en formación y definición. Hay una intensa y extensa tarea de difusión intelectual desde los roles de agentes culturales partícipes en el proceso de producción y circulación de escritos, autores, teorías, concepciones, debates, que exceden la inserción y reproducción en las instituciones académicas ${ }^{7}$.

Quizá en el caso de Ayala la etapa como partícipe directo en una institución universitaria es muy breve y bastante anómala dentro del exilio que vivió en Argentina. No obstante en los frentes intelectuales y

de México y Centro de Estudios Sociales; y (1947) Tratado de sociología. Buenos Aires, Losada.

$7 \quad$ Y, además, todas las charlas formales e informales, conversaciones casuales, las cenas, las visitas, las vacaciones, las cartas que se envían unos a otros; todo aquello va generando también puntos comunes de discusión, interés por autores e ideas que inevitablemente van enriqueciendo y modificando sus propios planteamientos. 
culturales de la edición y difusión su actividad fue prolífica. Sin la reposición de esta dimensión no se termina de comprender la importancia de su trayectoria durante el exilio en relación a la sociología, mientras que en el caso de Medina esta actividad ha sido bien estudiada (Blanco, 2008; Morales Martín, 2017; Morcillo Laiz, 2010; Moya López, 2013; Ribes, 2003). Esta pareciera ser otra diferencia que se advierte en la trayectoria institucional que cada uno de los sociólogos españoles realiza en México y Argentina.

En este sentido, por ejemplo, si se toma la colección dirigida por Medina siguiendo la cita de Moya López expresada con anterioridad, se muestra que los primeros libros responden a "la necesidad de proveer las bibliografías solicitadas por Medina Echavarría en sus cursos". En cambio, la vinculación de Ayala con las instituciones académicas fue mucho más intermitente. Su trabajo más formal estuvo enmarcado por un contrato profesoral en la Universidad Nacional del Litoral (UNL) entre 1941 y 1943 (Escobar, 2011, 2016a, 2017), tratándose de la mayor institucionalización en la trayectoria argentina del granadino. Todas las otras intervenciones fueron puntuales o bastante informales: como conferencista ocasional, algún seminario, participaciones como adscrito en el Instituto de Sociología de la Universidad de Buenos Aires (UBA), entre otras ${ }^{8}$.

Además, Ayala dictó varios seminarios y cursos breves en instituciones académicas y culturales de Argentina durante su estadía; algunas de ellas: en la Universidad Nacional del Litoral (UNL) en septiembre de 1940 sobre "El Estado liberal como objeto de conocimiento"; ; en el Ateneo Luis Bello (Ciudad de Rosario, Santa Fe) entre agosto y septiembre de 1942 bajo el título "Formas del Estado moderno" ${ }^{10}$; además,

\footnotetext{
$8 \quad$ A modo de citar ejemplos de lo expuesto, Ayala, en una carta enviada al secretario general Laudelino Ruiz del Ateneo Luis Bello (Ciudad de Rosario, Argentina), fechada 15 de agosto de 1942, narra que: "Mañana salgo para Tucumán, donde permaneceré algunos días". En la mencionada ciudad dictaría una conferencia en la Universidad Nacional de Tucumán (Archivo de la Fundación Francisco Ayala, Granada, España). Del mismo modo, en su libro Ensayo sobre la libertad (Ayala, 2009), el español da cuenta en el prólogo de que en agosto de 1943 expuso "su punto de vista sobre el problema de la libertad" en una conferencia en la Facultad de Derecho de la Universidad Nacional de Córdoba (p. 77).

9 Archivo Histórico y Museo de la Universidad Nacional del Litoral, Santa Fe (Argentina).

10 Archivo de la Fundación Francisco Ayala, Granada (España).
} 
en el Colegio Libre Estudios Superiores dictó algunos cursos entre 1940 y 1942 (Colegio Libre de Estudios Superiores, 1946). Asimismo, se pueden mencionar las actividades de 1945, año en que Ayala se asentó en Río de Janeiro, invitado y contratado por Benedicto Silva (funcionario del gobierno brasileño) como profesor de sociología para colaborar en la formación de jóvenes que ingresarían a la gestión pública brasileña. En dicha estadía también pronunció conferencias en Itamaraty, invitado por el ministro de Asuntos Exteriores, Leão Velloso, y en la Universidad de Río de Janeiro (García Montero, 2009).

Mientras que en el caso de Medina, desde su misma llegada estuvo vinculado al ámbito universitario mexicano en el cual desarrolló una tarea docente intensa hasta $1943^{11}$ (Morales Martín, 2017). En el citado año, Medina se hace cargo de la dirección del Centro de Estudios Sociales de El Colegio de México. En la lectura de Laura Moya López (2013),

el punto culminante de la trayectoria académica de Medina Echavarría en México fue la dirección del Centro de Estudios Sociales de El Colegio de México de 1943 a 1946, labor que se entrelazó con la dirección de la Colección de Sociología del Fondo de Cultura Económica y también con otras colecciones de esta casa editorial como lo fueron la de economía, historia y ciencia política. (p. 81).

Esta novedosa institución académica, constituida por las iniciativas de Cosío y Medina, basó su organización en el modelo de enfoques multidisciplinarios que había desarrollado la Universidad de Chicago en el área de las ciencias sociales y humanidades. Sería un proyecto que, aunque no prosperó en esta etapa, fue un intento importante e innovador en los planes de crear mayor institucionalización disciplinar en tanto propiciaba el avance de un centro de enseñanza e investigación en ciencias sociales (Blanco, 2008).

A partir de estas breves intromisiones en las trayectorias latinoamericanas de ambos exiliados, es posible aludir que la de Medina en México, sin dudas, fue más institucionalizada y académica que la de Ayala - aunque también con varios vaivenes-, pero más allá de las

11 El periodo intenso de docencia universitaria de Medina coincide con los asesoramientos y pedidos bibliográficos que hace corresponder y menciona Laura Moya López (2013, p. 93). de 1939 a 1943 en el catálogo de la SOS. 
diferencias, en ambos casos hay una relación permanente y que confluye en algunos fines, a pesar de las diferencias de cada situación.

Asimismo, es importante considerar que la sociología en la región latinoamericana aún era "una disciplina por entonces teóricamente poderosa pero institucionalmente débil” (Blanco, 2008, p. 4). Quizá, en parte, se deba a la situación general, que con más o menos avances y singularidades aún caracterizaba a la sociología $-\mathrm{y}$, por ende, a los sociólogos-, esto pareciera ser la de una precariedad institucional que logra saldarse en buena parte luego de la segunda guerra mundial. Desde el siglo XIX hasta la posguerra, no hay un lugar plenamente preciso y definido para los sociólogos, lo cual permite ver y explicar el trabajo difuso en varios frentes y a veces en posiciones precarias e inestables, como el caso de Georg Simmel, o directamente externas al mundo académico, como Marx o Saint-Simon, por nombrar casos de los más conocidos.

En consonancia con lo que venimos exponiendo, es imprescindible una lectura amplia que involucre espacios, producciones, estrategias, de los agentes intervinientes que buscan - desde posiciones casi siempre inestables o imprecisas- entretejer prácticas diversas para sostenerse e intentar consolidarse en campos en definición y, por ende, débiles que no permiten —en el mejor de los casos- institucionalizar y sostener plenamente las trayectorias. Y quizás es el fundamento de sus propias prácticas que no logran traducirse en una institucionalización consolidada lo que explique el abanico intenso y extenso (a veces sistemático y otras difuso) de sus producciones y variadas intervenciones intelectuales. Como planteamos, se torna preciso atender a varios frentes debido a que hay un margen mucho más grande que excede las prácticas académico-universitarias, y que puede ser tan potente y necesario como lo que se está constituyendo hacia dentro de los espacios académicos. Por lo cual, se observarán los circuitos y circulantes que contextualmente ubicados trabajan en diálogo y se relacionan mostrando un proceso más denso.

Hasta aquí, el caso de la edición es un primer paso en cuanto a producción que contiene a su vez la reinserción y validación a través de la puesta (y apuesta) en juego de los sociólogos exiliados en relación a campos débiles, pero en constitución real; pero una vez formado el circulante, a través de la producción editorial, cómo comenzó a 
circular y difundirse en los espacios académico-intelectuales y culturales.

Tanto Medina como Ayala, desde la posición de directores y asesores editoriales, fueron articuladores y dinamizadores claves de un circuito que entramaba diálogos de renovación disciplinar regional. Ambos gestaron y participaron en la articulación concreta de un corredor de ideas, a través del intercambio científico, editorial y epistolar, que se fue ramificando espacialmente y vehiculó una visión compartida de la sociología (Morales Martín, 2014). La estrecha relación entre ambos sociólogos exiliados es casi ininterrumpida a pesar de las distancias y desigualdades espaciales. Ambos retoman algunos de sus proyectos previos al exilio en la medida de las posibilidades reales de la región. A modo de ejemplo, entre los comentarios vertidos por Medina en su solicitud de beca a la Junta para Ampliación de Estudios de 1936, anterior a la Guerra Civil, en relación a su trabajo como editor y traductor en España de la Revista de Derecho Privado (RDP), decía que publicarían prontamente a Mannheim, Tönnies, Weber, Michels, Ruggiero, Lazarsfeld, Jehodn y Burns. De Mannheim, apareció en el catálogo de la RDP ese mismo año El hombre y la sociedad en la época de crisis con la traducción de Ayala ${ }^{12}$; luego, con el quiebre provocado por la guerra, el proyecto editorial no siguió, pero, en parte, esta proyección editorial sería retomada en el exilio latinoamericano.

\section{Mannheim como referencia}

El caso de Karl Mannheim (Budapest, 1893-Londres, 1947) quizá sea el más ilustrativo de la continuidad del proyecto editorial español truncado que, además, se incrusta en un universo más denso y profundo de ideas y problemáticas comunes. Del autor se publicaron tres títulos en la colección del FCE dirigida por Medina - y un cuarto libro editado en la SOS en 1953 y otro en 1963-, lo que lo posicionó como "el autor más editado y llegaría a convertirse en una especie de consejero informal de la editorial" (Blanco, 2009, p. 400), debido al intercambio que sostuvo con los agentes del FCE - en particular con Cosío Villegas-des-

Esta obra se editaría de nuevo, y con la misma traducción de Ayala, en Argentina bajo el sello editorial Leviatán en el año 1958. 
de 1939 hasta su temprano fallecimiento (Blanco, 2009; Garciadiego, 2016; Morales Martín, 2017; Moya López, 2013).

Los sociólogos exiliados tienen una sintomática influencia en relación a Mannheim, asociada a que el sociólogo húngaro leyó e interpretó la crisis de las sociedades modernas de su presente - tema y problema que articula y atraviesa los enfoques de los sociólogos exiliados-: "el conjunto de las esenciales mutaciones que experimentamos hoy, sólo puede ser captado con acierto cuando lo concebimos como la disolución de una forma anterior de la Sociedad y como el proceso de edificación de una nueva" (Mannheim, 1984, p. 11), en palabras del propio sociólogo. La primera traducción de Mannheim al castellano fue realizada, como anticipamos, por Francisco Ayala en 1936 y como presta atención Ribes (2007), la idea de crisis se hace presente hasta en pequeños detalles como en la traducción de su título, debido a que el "título original en su edición alemana era El hombre y la sociedad en la era de la reconstrucción (...) Ayala o los editores, decidieron sustituir 'era de reconstrucción', con las implicaciones evidentes que ese cambio comporta" (p. 131).

Por otra parte, al igual que los sociólogos españoles, Mannheim era un transterrado. Hijo de madre judía alemana y padre judío húngaro, sufre en su propia biografía las consecuencias de la convulsionada Europa de la primera mitad del siglo XX. En principio, huyó de Hungría en 1919 debido a las tempranas consecuencias de postguerra. Como bien presenta Lamo de Espinosa (1993), la revolución húngara de 1918 con la breve proclama y acciones de la República Soviética Húngara y la posterior represalia derechista desatada en 1919 por el Coronel Micklos Horthy le obligan a exiliarse, estableciéndose en Alemania, donde, en 1933, con el ascenso de Hitler y del nazismo fue expulsado una vez más para refugiarse en Londres, ciudad en la que vivió hasta su temprana muerte.

La trayectoria de Mannheim ${ }^{13}$ crea una afinidad electiva que da cuenta de las experiencias semejantes que comparten con Medina y Ayala (Escobar, 2018), que incluso va más allá de la sola inspiración académica. Los tres son exiliados; incluso se exilian en dos o tres ocasiones,

13 En contra de los nacionalismos étnicos y los fascismos, así como su fundamental condición de exiliado debido al antisemitismo que asola a Europa durante la primera mitad del siglo XX y que culmina en el horror del Holocausto. 
simbolizando, así, la compleja y delicada situación que atravesó la inteligencia y la suerte que sufrieron los intelectuales — pero también los judíos, los demócratas y otros tantos ciudadanos- a lo largo del siglo XX. A la par que Mannheim se enlaza con la tradición intelectual española de Ortega y Gasset, que junto a Adolfo Posada, fueron la gran referencia de estos sociólogos españoles -más allá de que casi siempre traten de ponerse en la vereda contraria-. Tanto la temática de la sociedad de masas, como de la crisis y también la categoría de generaciones (Ayala, 1944, 1946, 2009), fueron cuestiones típicamente orteguianas, posibilitando un cierto nexo Mannheim-Ortega, por lo cual hay una suerte de continuación del maestro Ortega junto con una afinidad intelectual, política y personal entre Mannheim y Ayala y Medina.

En la lectura de Ayala se hace evidente una relación con su propia definición de la sociología, complementándose de manera adecuada: "En su Ideología y utopía ha mostrado Karl Mannheim de manera magistral el proceso de 'desenmascaramiento' intelectual que fue el resultado de la gran crisis de donde la sociología había de surgir" (Ayala, 2008, p. 860).

En 1943 se edita Responsabilidad de la inteligencia: Estudios sobre nuestro tiempo, de Medina - bajo un título de evidente influjo mannheniano-, mientras que al siguiente año Ayala publica Razón del mundo. Un examen de conciencia intelectual, cuyo primer capítulo se denomina "La responsabilidad de la inteligencia". En ambos libros hay problemáticas recurrentes atravesadas por las responsabilidades del intelectual, igual que su desprestigio en la sociedad contemporánea: la sociedad de masas, que sustituye a la sociedad clasista (Ayala, 2008). La coincidencia no solo está en el tema, sino también en las fechas. Por otra parte, los dos ensayos son compilaciones de artículos ya publicados; en el caso de Medina, "Responsabilidad de la inteligencia", que da título a su libro, se publicaba en junio de 1941 en el Noticiero Bibliográfico como comentario sobre la aparición del libro de Mannheim Ideología y utopía; mientras que Ayala divulgaba el artículo "Examen de conciencia. La responsabilidad de la inteligencia" en el diario La Nación del 9 de noviembre de $1941^{14}$ a partir del mismo libro del sociólogo húngaro.

Algunos fragmentos de este artículo se encuentran en la introducción y en el capítulo del mismo título de Razón del mundo (Ayala, 1944). 
Parece que los tres se toman muy en serio la responsabilidad que consideran tener como intelectuales y sociólogos en un momento en el que Europa ha estallado en llamas. La defensa de un compromiso académico y profesional con el presente es otro de sus nexos que señalan los tres con ese guiño, sin duda, intencionado que supone el rótulo de responsabilidad de la inteligencia; aparece una lectura subterránea en la cual se traduce un cierto deber de hacerse cargo de esa situación, porque no hacerlo o dejarse llevar por el irracionalismo que asolaba Europa, era una clara traición a su posición política, académica y a su forma de entender su papel en el mundo.

El tema sobre el que giran ambos libros — con coincidencias pero también con importantes diferencias - ya aparecía planteado en sus líneas generales en El hombre y la sociedad en la época de crisis (Mannheim, 1984). Allí, Mannheim advertía que una de las consecuencias de la "sociedad democrática de masas" es la "proletarización de la intelectualidad" (Mannheim, 1984, p. 84), debido a que "el exceso de oferta de intelectuales hace caer el valor de los intelectuales", pero el verdadero problema que esto porta no es la "desvalorización de las profesiones espirituales" en sí, sino la "desvalorización del espíritu mismo en la opinión pública” (p. 85). En sintonía con Max Weber, pero también con Marx, Mannheim argumentaba sobre las relaciones del pensamiento y de la reflexión con la realidad social vital, en donde el pensamiento se expresa, la vinculación entre existencia social concreta y su conciencia derivada.

En esta línea Ayala reflexiona que "todo pensamiento responde a un complejo de circunstancias, a una determinada situación histórica, y que sirve a hondas exigencias sociales derivadas de esa situación" (Ayala, 2009, p. 344). La traducción de Ideología y utopía, dirá Ayala (2009), en tanto "pequeño renacimiento (...), llega a nosotros cuando nos ocupa el tema de la responsabilidad de los intelectuales: a la hora de nuestro examen de conciencia" (pp. 300-301).

El sociólogo español reflexionará — sobre todo en la primera parte del libro- respecto de la situación del intelectual en la sociedad de masas, prestando atención al contexto del debate de época, para lo cual parte de la metáfora de que el intelectual constituye la conciencia del cuerpo social, y en su contexto actual de escritura, los tiempos situados "son tiempos de examen de conciencia" (Ayala, 2009, p. 
298). Posteriormente se adentra en los argumentos para construir y postular una imagen del intelectual: "es la del hombre que contempla el mundo, y lo piensa" (p. 335), y ahí hay, precisamente, otro momento mannheiniano, ya que "existe una vinculación entre el pensamiento y la realidad social, de la que aquel es función" (p. 345); y la situación de su presente está atravesada y se define por la crisis, que atenaza la conciencia intelectual cuestionando su lugar y función. Situación presente de crisis, conciencia y pensamiento: allí está un enfoque sociológico en el cual Mannheim se aúna en una sociología, en tanto conciencia de la realidad que responde al inmediato presente. Los intelectuales deben

esforzarse sin descanso por hallar, en medio de la crisis y a favor de su coyuntura, el sentido de la realidad histórica en que se encuentran implicados y, desde el centro de esa realidad, pensar los temas eternos con sinceridad implacable; mantener viva, en incansable clamor, la demanda por el destino esencial del hombre. (Ayala, 2009, p. 411).

Esta es, en parte, una respuesta ayaliana que conjuga el enfoque sociológico con una condición humana siempre muy presente en una salida ética, un ethos para atravesar la crisis, y es, a la vez, una diferencia directriz frente a Mannheim y también con Medina Echavarría. Mientras que para ellos la salida propuesta - a grandes rasgos- consiste en más racionalidad (por ejemplo, a través de la planificación y la ciencia), para Ayala la salida la encuentra precisamente en una posible alternativa ética, que reconstruya y restituya un nuevo ethos a la modernidad —enfoque, a su vez, atravesado por Max Weber- (Escobar \& Ribes, en prensa).

El argumento de Alejandro Blanco (2009) es muy acertado al destacar la importancia que tuvo Karl Mannheim a partir de la década del cuarenta, cuando comenzó a estar "en boca de muchos intelectuales, pensadores sociales y sociólogos de América Latina”, mientras que de manera previa era un sociólogo inexistente en referencias y tradiciones locales (Blanco, 2009, pp. 393-394). Sin duda que el trabajo de traducción y de difusión de los sociólogos exiliados es una de las puntas de fundamental importancia para comprender la introducción del sociólogo húngaro. 


\section{Tönnies y Weber a la luz del exilio}

Por otro lado, los otros dos autores retomados del proyecto inconcluso de la RDP son quizás los que tenían una introducción previa bastante más firme; estamos hablando de Ferdinand Tönnies y Max Weber.

Sobre el primero, Ayala publica en 1941 un artículo titulado "El concepto sociológico de nación”, allí retoma y amplía el binomio comunidad-sociedad de Tönnies para plantear y cuestionar la relación de nación y Estado; incluso dentro del catálogo que dirigió Ayala, la Biblioteca Sociológica, se traduce y publica Comunidad y sociedad de Tönnies (editorial Losada, 1947). Mientras que la SOS a cargo de Medina incorporaría a su catálogo Principios de sociología de Tönnies (Fondo de Cultura Económica, 1942, traducción de Vicente Lloréns).

Ayala trabaja en sus escritos con tres obras de Tönnies, mencionadas de forma explícita: Comunidad y sociedad (Leipzig, 1887; trad. esp. 1947), Introducción a la sociología (Stuttgart, 1931) y Kritik der öffentlichen Meinung (Berlín, 1922). De los tres títulos, el más citado y abordado es el primero que en conjunto con el segundo serán trabajados en forma específica en su Tratado de sociología, bajo el subtítulo "Construcción del sistema. Categorías propuestas por F. Tönnies. La solución viable" (2008) ${ }^{15}$; mientras que Kritik der öffentlichen Meinung será propuesta como "el esfuerzo más considerable realizado desde el campo de la sociología" sobre el estudio de la opinión pública, el que fuera llevado a cabo "en plena crisis social y en un país, Alemania, (...) donde la concepción de un gobierno de opinión pública había sido siempre tenida en jaque por concepciones autoritarias del Estado" (Ayala, 2009, p. 217).

Este último libro de Tönnies es considerado por Ayala, junto con los libros del norteamericano Lawrence Lowell (Public Opinion and Popular Government) y de otro húngaro, Ernst Manheim (Die Träger der

\footnotetext{
15 El interés de Ayala en el estudio de la opinión pública se debe a la búsqueda para comprender cómo es posible lo que estaba sucediendo en Europa, mientras que quizá también para prevenir futuras irrupciones del irracionalismo que pongan en jaque de nuevo las libertades individuales tan defendidas en Ayala. La opinión pública y la manipulación de la misma también había sido un tema central en Mannheim, dada su importancia crucial en el régimen nazi. Ayala, aquí, está defendiendo, como siempre, la libertad de la mejor manera que puede, es decir, estudiando o prestando atención a algún aspecto, en este caso, a la opinión pública.
} 
öffentlichen) -título sobre el que acota en relación a la edición española de 1936, "que yo traduje hace unos años" (Ayala, 2009, p. 217-, como los únicos aportes importantes sobre el estudio de la opinión pública. Debido a que el resto de "estudios de la opinión pública que integran la bibliografía del tema (...) no alcanzan a superar el estadio de digresiones sagaces pero parciales, o de una profundización sobre los caracteres y condiciones del régimen político liberal" (Ayala, 2009, p. 217).

Por supuesto que no son las únicas veces en que Tönnies es incorporado a los escritos de Ayala. Desde el primer momento, en el capítulo I del Tratado..., es mencionado junto a Simmel, en sentido estricto, como los tardíos primeros sistematizadores de la sociología en Alemania - en la cual lee, como fuentes primeras, la filosofía de Hegel y las obras de von Stein y Marx-. Asimismo, es principio de diferencias, comparaciones y matices con las interpretaciones de Oppenheimer sobre la culminación del "proceso social o histórico" como unidad (Ayala, 2009) ${ }^{16}$.

En tanto que Max Weber constituye uno de los casos de recepción y difusión que ha generado varios estudios en la región, considerando solo algunas de las principales investigaciones recientes (Aronson \& Weisz, 2007; Blanco, 2007; Morales Martín, 2017; Morcillo Laiz, 2012; Morcillo Laiz \& Weisz, 2016; Moya López, 2013). El temprano ingreso de Max Weber, junto con nuevas traducciones, ha llamado la atención de investigadores sociales debido a que "este proceso es excepcional en cuanto a la secuencia de las traducciones, a la recepción temprana combinada con la ausencia de institucionalización en programas de estudio y en una o varias escuelas de sociólogos y científicos políticos" (Morcillo Laiz \& Weisz, 2016, p. 21).

Sin dudas que la titánica traducción de Economía y sociedad (1944), se convirtió en la obra que distinguió al catálogo de FCE de manera muy temprana, colocándolo en punta dentro de las traducciones del autor alemán a las lenguas latinas e incluso anglosajonas, ya que, de manera íntegra, era la primera edición fuera de la lengua alema-

${ }_{16}$ Si bien la obra traducida y publicada de Tönnies en la SOS del FCE dirigida por Medina no es trabajada explícitamente, Ayala la nombra dentro del Nomenclátor biobibliográfico de la sociología que acompaña, como tomo III, al Tratado de sociología en su primera edición (Ayala, 1947). 
na (Morcillo Laiz, 2012); traducción a la que ya le había precedido en 1942, también en la SOS, Historia económica general (traducción de Manuel Sánchez Sarto).

Sin embargo, los sociólogos españoles son una vez más una pieza clave en el entramado, ya que para ellos se trataba de un autor en el que se interesaron desde su etapa formativa en Alemania - aunque apenas llegaron a divulgarlo en su tierra de origen-. Ya en el exilio, se volverían una pieza clave en la difusión y fortalecimiento del pensamiento de Weber en la región, "a partir del corredor de ideas establecido entre Argentina y México” (Morales Martín, 2017, p. 610), como lo muestran sus intercambios epistolares, sus obras y escritos para revistas y diarios, entre otras acciones.

Sobre la aparición de Economía y sociedad en el FCE, Ayala escribía a Medina:

Ya he visto los dos primeros tomos de Economía y sociedad. Es toda una empresa publicar un libro así; te felicito. He publicado un primer artículo en La Nación comentando el libro, y seguirá otro que estoy haciendo para el mismo periódico. ${ }^{17}$

Este enlace muestra la tarea editorial por fuera del mundo específicamente editorial, ya que se trata de escritos para la prensa periódica de tirada masiva, instalándola frente a una recepción bien amplia en el espacio público de Argentina. Tanto Ayala como Medina, cada uno en la medida de las posibilidades que operaban en los medios directos en los que se movían, no dejaron de indagar e introducir a Max Weber con reseñas, discusiones y reflexiones (Escobar \& Ribes, 2020). Por otra parte, es importante considerar que esta difusión intelectual se realiza en un contexto de traslado transatlántico de la interpretación weberiana, puesto que "desde 1945 la hegemonía de la sociología norteamericana conllevó también la pérdida del carácter histórico de lo social en aras de una teoría sistemática de la sociedad" (Morcillo Laiz y Weisz, 2016, p. 25).

La proyección editorial de la RDP sería retomada en el exilio y rearticulada bajo las nuevas condiciones mediante el corredor de ideas,

17 Carta de Francisco Ayala a José Medina Echavarría (20/11/1944). Archivo de la Fundación Francisco Ayala, Granada (España). 
que se fue intensificando y extendiendo en sus ramificaciones espaciales a partir de la comunicación y las acciones concretas entre Medina Echavarría y Ayala, así como los espacios institucionales y culturales en los que se movieron y los agentes implicados en ellos.

\section{Breves anotaciones sobre Dewey}

Con el fin de cerrar con una acotada ejemplificación de cómo el corredor de ideas se iba ramificando, traeremos algunas vinculaciones a partir de un autor: John Dewey.

En El Trimestre Económico (Vol. 6, Nㅜㄹ) correspondiente a eneromarzo de 1940, Medina Echavarría publicaba un ensayo sobre el libro de John Dewey que hacía poco tiempo había aparecido en Estados Unidos, Freedom and culture (1939). Allí abre la escritura argumentando que una vigilancia perpetua es el precio de la libertad, tarea que concierne a todos los hombres, y "John Dewey no ha rehuido esa responsabilidad (...) fiel a sí mismo indaga cuál es la 'experiencia' de estos tiempos para la vida de la democracia” (Medina Echavarría, 1940, p. 613). Este escrito sería incluido en Responsabilidad de la Inteligencia: Estudios sobre nuestro tiempo (Medina, 1943) ${ }^{18}$. Siguiendo los argumentos de Ramón del Castillo Santos, este escrito de Medina se trata de una

pieza de enorme profundidad y muy por encima de lo que muchos comentaristas de Dewey han dicho sobre el tema. Con sólo leer esta breve reseña, queda claro que Medina comprende a Dewey como pocos intelectuales de su época y como ningún otro pensador en lengua española. Sobresale en su interpretación, sin duda, el lado más social del pensamiento de Dewey. (Castillo Santos del, 2014, p. 137).

18 Dewey es un autor que Medina comenzaría a introducir en este contexto en programas, cursos y en sus libros (Morales Martín, 2017). Más allá de diversos hechos que fueron determinando el rumbo de la difusión de las obras de Dewey en México (Castillo Santos del, 2014), el FCE incorporaría formalmente a Dewey en su colección de filosofía a partir de un primer título publicado en 1948: La experiencia y la naturaleza (traducción de José Gaos); luego, seguiría, en 1949, El arte como experiencia (traducción y prólogo de Samuel Ramos); en 1950, Lógica: teoría de la investigación (traducción y prólogo de Eugenio Imáz); y, en 1952, La busca de la certeza (traducción de Eugenio Imáz). Lo que sí es pertinente agregar es que

sin la influencia de Medina, la recepción de Dewey en México habría sido muy distinta. (...) Medina fue el primero que consideró relevante la traducción de obras de Dewey en el FCE y quien emprendió negociaciones de derechos para llevar a cabo las traducciones. (Castillo Santos del, 2014, pp. 136-137). 
El texto reseñado de Dewey estuvo dentro de las consideraciones a traducirse y publicarse en el FCE en ese mismo año de común acuerdo entre Medina y Cosío Villegas - a su vez recomendado, en tanto autor, por Mannheim-. Sin embargo, debido a cuestiones económicas y en particular a otras situaciones bien abordadas en el trabajo de Ramón del Castillo Santos (2014), la posibilidad de publicación quedó abandonada en los términos propuestos por Medina. Finalmente, se editarían otros títulos de John Dewey años más tarde dentro del catálogo de filosofía de la editorial mexicana y sin la participación de Medina.

A pesar de que desde Argentina Francisco Ayala no abordó de manera directa las obras del autor estadounidense en su literatura ${ }^{19}$, sin embargo, John Dewey aparece reseñado en el Nomenclátor bio-bibliográfico de la sociología del Tratado de sociología (Ayala, 1947). Allí, cuando se busca en la letra $D$ en esta suerte de diccionario, se encuentra, junto a una brevísima reseña biográfica de Dewey, un listado de quince obras y dos traducciones al español. Quien traduce al español el primer libro editado en Argentina al español de John Dewey en el año 1946 es, ni más ni menos, que la discípula predilecta de Francisco Ayala en Argentina: Ángela Romera Vera ${ }^{20}$. Ella había oficiado de mediadora en la UNL para el ingreso del sociólogo español a la cátedra de sociología en su breve paso universitario. Además, Romera Vera integró el grupo de investigación que dirigió Ayala en este espacio institucional, a la par que participó, en este período estudiado, en casi todos los espacios intelectuales y culturales con los que estuvo vinculado Ayala (Escobar, 2016b).

El título, que se publica por la editorial Rosario - espacio, a su vez, relacionado a los circuitos externos en la región de la UNL-, es, justamente, Libertad y cultura. Una vez más esta nueva acción concerta-

19 Las menciones directas a Dewey aparecen recién en 1958 en artículos de Ayala sobre la educación, cuando se encuentra en Estados Unidos como profesor en la Universidad de Princeton.

20 Ángela Romera Vera (Córdoba, 1912 - Santa Fe, 1990) realizó sus estudios en España. A mediados de 1930 se licenció en Derecho en la Universidad Complutense de Madrid. Hacia 1936, con el inicio de la Guerra Civil, regresó a la Argentina y se estableció de manera definitiva en la ciudad de Santa Fe; revalidó su título en la Facultad de Ciencias Jurídicas y Sociales de la UNL, doctorándose en 1940 con la tesis titulada "Estado y Derecho", bajo la dirección de Francisco Ayala. Romera Vera será artífice de muchos de los vínculos intelectuales y culturales de Ayala en Argentina, amiga y discípula y, a la vez, es una gestora institucional de la sociología en la UNL en el periodo posterior al peronismo (Escobar, 2016b). 
da, o coincidencia de intereses, vuelve a simbolizar la necesidad de la defensa de la razón, de la ciencia, de la pluralidad y de la democracia. Dewey aquí opera como un autor que encarna bien lo que tanto Mannheim como Medina y Ayala estaban tratando de reconstruir, sobre lo que querían advertir y lo que temían que pudiera extenderse por el mundo.

Así lo expresa Alejandro Blanco, refiriéndose a los debates que surgían alrededor de la democracia, el lugar de los intelectuales, las libertades, entre otros, que eran las grandes preocupaciones de la intelectualidad antifascista local:

en algunos textos de esos años, pero especialmente en Freedom and Culture, traducido por una editorial rosarina en 1946, Dewey (...) había puesto de relieve algo así como el código moral de la ciencia caracterizado por una disposición favorable a conservar las creencias en suspenso y a mantener la duda hasta el logro de la evidencia; a llegar hasta donde la evidencia apunta en vez de quedarse con una conclusión dictada por una preferencia personal y a emplear las ideas como hipótesis sujetas a prueba antes que como afirmaciones dogmáticas. (Blanco, 2006b, pp. 18-19).

De este modo, es posible observar de manera escueta cómo ese flujo de intercambios entre Medina y Ayala va creando ramificaciones concretas en una territorialidad ampliada que constituye el anclaje de un diálogo y de las difusiones intelectuales. Tanto la publicación sobre Freedom and culture de Dewey en México por Medina Echevarría como la traducción al español realizada por Romera Vera en Argentina dan cuenta de las sintonías de tales vinculaciones y materialidades que se consolidan en estos intercambios.

\section{A modo de conclusión}

Al considerar la propuesta que indaga el papel fundamental que jugaron Medina y Ayala en difusión de algunas obras, autores y de discusiones que, además de divulgar, incorporan en sus obras de una manera dialógica desde un intercambio ininterrumpido - no solo entre ambos sino también con su propias tradiciones y al agregar también a los sociólogos latinoamericanos a través de la actividad editorial, de las conferencias, las clases, etc.-, buscamos mostrar 
cómo la aclimatación y la difusión vinculada y concertada (o coincidente) tiene lugar en varios momentos y espacios que hemos querido ejemplificar, fundamentalmente, con la incorporación, divulgación y presentación de obras de Weber, Tönnies, Mannheim y, de forma bien breve, de Dewey.

Este trabajo muestra varias de las tareas de producción y difusión, como además, las fragilidades atadas a las trayectorias y los espacios en constitución y algunos vínculos en los nudos de las conexiones que los sociólogos exiliados españoles establecieron entre México y Argentina. Aquí se empiezan a articular un corredor de ideas, mediaciones y proyecciones que generan una ramificación espacial más densa y es sobre estas intervenciones donde la sociología comienza a re-estructurarse y, sobre todo, a potenciar las relaciones a nivel regional. Esto es visible en el intercambio epistolar, en los asesoramientos y directrices editoriales, en las proyecciones y trabajo sobre obras y autores, en la divulgación en medios escritos, en la vinculación con agentes de los campos culturales y académico-intelectuales, en la circulación de las obras de los catálogos, en sus propios escritos y debates, pero también en aquellos espacios cuyas huellas son de más difícil registro, pero de principal importancia: los cursos, clases, conferencias, charlas formales e informales, etc. Todas estas variadas acciones, que en mayor o en menor medida se han tratado de presentar en el artículo, fueron configurando ese corredor de ideas entre las dos capitales nacionales que, por el tenor mismo de las intervenciones y las circulaciones, se fueron ramificando desde cada acción a partir de los propios vínculos locales y regionales de los sociólogos españoles.

También, de forma un poco más indirecta dentro del escrito, se deja entrever a través del intercambio cómo esos dos viejos amigos de una España republicana derrotada, quienes habían querido creer en un futuro de progreso y democracia, y que habían confiado en las posibilidades emancipadoras de la disciplina sociológica, van entrecruzando sus peripecias vitales e intelectuales, sus exilios y sus inquietudes intelectuales, sus experiencias personales; y cómo, a pesar de las distancias, mantienen siempre una relación singular, buscando comprender sus experiencias y otorgarle sentido desde los propios escritos y lecturas. Allí aparece el compromiso político e intelectual ineludible con el presente de estos sociólogos exiliados. 
Como todo trabajo, lejos de cerrar, solo expone parcialidades siempre dinámicas y abiertas a la reciprocidad de la crítica y las nuevas búsquedas. La pesquisa de fondo, a veces más arrogante aunque casi siempre más dócil, continúa siendo la misma propuesta: abrir —en términos wallersteinianos- las cartografías existentes de la sociología en Latinoamérica, para revisar e incluir trayectorias, aportes y redes poco o nada visibilizadas.

\section{Referencias}

Archivo de la Fundación Francisco Ayala, Granada, España.

Archivo histórico y Museo de la Universidad Nacional del Litoral, Santa Fe, Argentina.

Aronson, P. \& Weisz, E. (Eds). (2007). La vigencia del pensamiento de Max Weber a cien años de "La ética protestante y el espíritu del capitalismo". Buenos Aires: Gorla.

Ayala, F. (1941). El concepto sociológico de Nación. Revista Universidad, (9), 187-224.-

Ayala, F. (1944). Planteamiento del problema de la generación como concepto sociológico. Boletín del Instituto de Sociología, (3), 51-55.

Ayala, F. (1946). Presentación. En W. Pinder, El problema de las generaciones en la historia del arte de Europa. (pp. 7-8). Buenos Aires: Losada.

Ayala, F. (1947). Nomenclátor bio-bibliográfico de la sociología. (Vol. 3. Tratado de sociología). Buenos Aires: Losada.

Ayala, F. (2008). Sociología y ciencias sociales (Vol. 4, Obras Completas). Barcelona: Galaxia Gutemberg.

Ayala, F. (2009). Ensayos políticos y sociológicos (Vol. 5, Obras Completas). Barcelona: Galaxia Gutemberg.

Blanco, A. (2006a). Razón y modernidad. Gino Germani y la sociología en la Argentina. Buenos Aires: Siglo XXI.-

Blanco, A. (2006b). Gino Germani: La renovación intelectual de la sociología. Buenos Aires: Universidad Nacional de Quilmes.

Blanco, A. (2007). La temprana recepción de Max Weber en la sociología argentina (1930-1950). Perfiles Latinoamericanos, (30), 9-38.

Blanco, A. (2008). José Medina Echavarría y el proyecto de una sociología científica. Trabajo presentado en V Jornadas de Sociolo- 
gía de la Universidad Nacional de La Plata. Facultad de Humanidades y Ciencias de la Educación, La Plata, Argentina. Recuperado de http://www.memoria.fahce.unlp.edu.ar/ trab_eventos/ev.5894/ev.5894.pdf

Blanco, A. (2009). Karl Mannheim en la formación de la sociología moderna en América Latina. Estudios Sociológicos, (80), 393-431.

Carta de Francisco Ayala a José Medina Echavarría (20/11/1944). Archivo Fundación Francisco Ayala, Granada, España.

Castillo Santos del, R. (2014). Pobres diablos: José Gaos, John Dewey y la metafísica made in USA (primera parte). Diánoia, 59 (72), 131-153.

Colegio Libre de Estudios Superiores. (1946). Quince años de labor 1930-1945. Buenos Aires: Talleres gráficos de Emilio Bustos.

Diego de, J. L. (2014). 1938-1955. La "época de oro" de la industria editorial. En J. L. de Diego (Dir.), Editores y políticas editoriales en Argentina (1880-2010) (pp. 97-133). Buenos Aires: Fondo de Cultura Económica.

Escobar, L. A. \& Ribes, A. J. (2020). El Max Weber del exilio republicano en las ciencias sociales latinoamericanas. Políticas de la $\mathrm{Me}$ moria, (en prensa).

Escobar, L. A. (2011). Francisco Ayala y la Universidad Nacional del Litoral. La construcción de una tradición sociológica. Granada: Universidad de Granada y Fundación Francisco Ayala.

Escobar, L. A. (2016a). La cátedra de sociología de la Facultad de Ciencias Jurídicas y Sociales. En G. Sozzo, G. (Dir.). Hacer derecho. Reconstrucciones acerca de la relación derecho/ciencias sociales en la FCJS de la UNL (pp. 185-203). Santa Fe: Universidad Nacional del Litoral.

Escobar, L. A. (2016b). Retazos de una tradición olvidada: Ángela Romera Vera. En G. Sozzo, G. (Dir.). Hacer derecho. Reconstrucciones acerca de la relación derecho/ciencias sociales en la FCJS de la UNL (pp. 125-141). Santa Fe: Universidad Nacional del Litoral.

Escobar, L. A. (2017). Proyectando una sociología latinoamericana: El Boletín del Instituto de Sociología de la Universidad de Buenos Aires y Francisco Ayala. Revista Temas Sociológicos, (21), pp. 119-147. 
Escobar, L. A. (2018). Redes latinoamericanas y exilio español en la construcción de una tradición sociológica. Francisco Ayala, sociólogo sin sociedad (Tesis doctoral inédita). Centro de Estudios Avanzados, Córdoba, Universidad Nacional de Córdoba.

Fondo de Cultura Económica. (2009). Catálogo histórico 1934-2009. México D. F.: Fondo de Cultura Económica.

García Montero, L. (2009). Francisco Ayala. El escritor en su siglo. Granada: Diputación Provincial de Granada.

Garciadiego, J. (2016). El Fondo, La Casa y la introducción del pensamiento moderno y universal al español. México D. F.: Fondo de Cultura Económica.

Lamo de Espinosa, E. (1993). Introducción. En el centenario de Karl Mannheim (1893-1947). Revista Española de Investigaciones Sociológicas, (62/93), 7-13.

Mannheim, K. (1984). El hombre y la sociedad en la época de crisis. Buenos Aires: Leviatán.

Medina Echavarría, J. (1940). John Dewey y la libertad. En Trimestre Económico, (24), 613-624.-

Morales Martín, J. (2014). Un corredor de ideas entre México y Argentina. El intercambio científico e intelectual entre los sociólogos del exilio español. Iberoamericana, (53), 29-48.

Morales Martín, J. (2017). José Medina Echavarría: vida y sociología. México D. F.: El Colegio de México.

Morcillo Laiz, A. \& Weisz, E. (Eds). (2016). Max Weber en Iberoamérica. México D. F.: Fondo de Cultura Económica.

Morcillo Laiz, A. (2010). El forastero que se queda: José Medina Echavarría y la sociología latinoamericana. En M. González Navarro, A. Castañón y A. Morcillo Laiz (Ed.), José Medina Echavarría y México (pp.343-372). México D. F.: El Colegio de México.

Morcillo Laiz, A. (2012). Aviso a los navegantes. La traducción al español de Economía y sociedad de Max Weber. Estudios Sociológicos 30, (90), 609-640.

Moya López, L. (2013). José Medina Echavarría y sociología como ciencia social concreta (1939-1980). México D. F.: El Colegio de México.

Ribes, A. J. (2003). Presentación. La sociología de José Medina Echavarría (1903-1977) en el centenario de su nacimiento: Teoría 
sociológica, divulgación y sociología del desarrollo. Revista Española de Investigaciones Sociológicas, (102/03), 263-272.

Ribes, A. J. (2007). Paisajes del siglo XX. Sociología y literatura en Francisco Ayala. Madrid: Biblioteca Nueva.

Sorá, G. (2010). Misión de la edición para una cultura en crisis. El Fondo de Cultura Económica y el americanismo en tierra firme. En C. Altamirano, (Dir.), Historia de los intelectuales en América Latina II (pp 537-566). Buenos Aires: Katz Editores. 